\title{
The Effects of Wrong Scale Factor on the Phases and the Electron Density Computed by the Anomalous Dispersion Method
}

\author{
BY A. K. SINGH \\ Material Sciences Division, National Aeronautical Laboratory, Bangalore-17, India
}

(Received 14 July 1966 and in revised form 22 September 1967)

\begin{abstract}
The effects of a wrong scale factor on the phases computed by the anomalous-dispersion method and on the resulting electron-density map have been considered. The errors introduced in the phases are functions of $\left|\alpha(H)-\alpha_{A}(H)\right|$ and the scale factor, $\alpha(H)$ and $\alpha_{A}(H)$ being the correct phase and the heavyatom phase. The electron density computed with these phases can be expressed as a sum of a number of component syntheses of which one is very similar to the correct electron density and another to the synthesis based on the heavy-atom phases. The remaining terms contribute mainly to the background. As a result the electron density computed with a wrong scale contains the structure on an enhanced background. The results have been verified in an example based on an actual case.
\end{abstract}

\section{Introduction}

That the differences in the intensities of inverse reflexions, when an anomalous scatterer is present in a noncentrosymmetric crystal, can be used to determine its structure was suggested by Okaya \& Pepinsky (1956), Peerdeman \& Bijvoet (1956) and Ramachandran \& Raman (1956). Since then the method of phase determination (Ramachandran \& Raman, 1956), has been successfully used by many workers such as Geurtz (1963), Dale, Hodgkin \& Venkatesan (1963), Hall \& Maslen (1965), and Nockolds (1966). The application of this method needs the intensity data on an absolute scale which is not known accurately in the initial stages of the structure analysis. The scale factor determined by Wilson's (1942) method may be wrong by 40 to $50 \%$. Thus any error in the scale factor will show itself in the phases. As the phases seem to be more important than amplitudes (Srinivasan, 1961) in structure analysis, the resulting electron density map is expected to be affected adversely. However, in spite of this some investigators (Geurtz, 1963; Hall \& Maslen, 1965) have demonstrated in specific cases the insensitiveness of the electron density to the errors in scale factor.

In the present communication the effects of a wrong scale factor on the phases and the resulting electrondensity map have been considered. It is found that the error introduced in the phase of any reflexion depends on $\left|\alpha(H)-\alpha_{A}(H)\right|$ and the magnitude of the scale factor; $\alpha(H)$ and $\alpha_{A}(H)$ denote the correct phase and the heavy-atom* phase.

The nature of the electron density computed with these phases can be described as follows.

The electron density computed with data on a scale lower than absolute can be represented as the sum of five component syntheses, two of which are the correct

\footnotetext{
* Since the anomalous scatterers are invariably 'heavy atoms', the expressions 'heavy atom' and 'anomalous scatterer' have been used here interchangeably.
}

electron density and the synthesis with heavy-atom phases. The other components provide background. As a result the electron density with wrongly scaled data contains the structure superimposed on a background.

In case the data are on a scale higher than absolute, the electron density can be expressed as the sum of six component syntheses. For the various reasons discussed in the paper, the interpretation of the component syntheses becomes difficult. However, if the error in the scale factor is not large, two of the six component syntheses are very similar to the correct electron density and the synthesis based on the heavy atom phases. As a result electron density computed with a wrong scale contains the structure on an increased background.

The intensity data may contain a group of centrosymmetric reflexions. Such reflexions have, the heavy atom phase (neglecting a few cases where the phase of the reflexion differs from the heavy atom phase by $\pi$ ) and are not altered by the errors in scale factor. In the present analysis such reflexions have not been considered. However, it can be stated qualitatively that the presence of such reflexions will make the electron density less sensitive to the errors in scale factor.

To verify the results obtained in this paper, data based on the $(001)$ projection of ephedrine hydrochloride (Phillips, 1954) have been used. The agreement between the theory and the actual computations is good.

\section{The method of phase determination}

In this section we shall briefly outline the method of phase determination and discuss some of the aspects which will be used in the subsequent analysis.

Ramachandran \& Raman (1956) have shown that the phase $\alpha(H)$ of $F^{\prime}(H)$ is related to the phase of the anomalous scatterer $\alpha_{A}(H)$ by

$$
\alpha(H)=\alpha_{A}(H)+\pi / 2 \pm \theta,
$$


$0 \leq \theta \leq \pi$ and $\theta$ is given by

$$
\cos \theta=\Delta F^{2} / 4\left|F^{\prime}(H)\right|\left|F_{A}^{\prime 2}(H)\right|,
$$

where $\Delta F^{2}=\left[|F(H)|^{2}-|F(\bar{H})|^{2}\right],\left|F^{\prime}(H)\right|=\left[\frac{1}{2}\left\{|F(H)|^{2}\right.\right.$ $\left.\left.+|F(\bar{H})|^{2}\right\}-\left|F_{A}^{\prime \prime}(H)\right|^{2}\right]^{1 / 2}$ and $\left|F_{A}^{\prime \prime}(H)\right|$ is the contribution of the anomalous scatterer corresponding to the imaginary component of the scattering factor. The limits $0 \leq \theta \leq \pi$ arise from the convention of choosing $\theta$ between 0 and $\pi / 2$ or $\pi / 2$ and $\pi$ according as $\Delta F^{2}$ is positive or negative. Equations (1) and (2) are valid when all the anomalous scatterers are identical.

Thus for $0 \leq\left|\alpha(H)-\alpha_{A}(H)\right|<\pi / 2$ the phases are given by

$$
\alpha_{1}(H)=\alpha_{A}(H)+\pi / 2-\theta,
$$

and for $\pi / 2<\left|\alpha(H)-\alpha_{A}(H)\right| \leq \pi$ the phases are given by

$$
\alpha_{2}(H)=\alpha_{A}(H)+\pi / 2+\theta .
$$

Thus there exists a twofold ambiguity in the determination of the phases. This ambiguity is usually resolved by choosing from the two possible solutions, the one closer to $\alpha_{A}(H)$. It is readily seen that $\alpha_{1}(H)$ is closer to $\alpha_{A}(H)$ than $\alpha_{2}(H)$ since $\left|\alpha_{1}(H)-\alpha_{A}(H)\right|<$ $\left|\alpha_{2}(H)-\alpha_{A}(H)\right|$. The success of this method of resolving the phase ambiguity indicates that for most reflexions $\left|\alpha(H)-\alpha_{A}(H)\right|$ lies between 0 and $\pi / 2$. The theoretical studies on the distribution of $\left|\alpha(H)-\alpha_{A}(H)\right|$ by Parthasarathy (1965) support this fact. The distribution of $\left|\alpha(H)-\alpha_{A}(H)\right|$ depends on the heavy atom ratio $\sigma^{2}\left(=\sum f_{A}^{2} / \sum f_{n}^{2}\right)$ and on the number of anomalous scatterers and their arrangement in the unit cell. However the nature of the distribution does not change markedly (Fig. 3 of Parthasarathy, 1965) with the num-

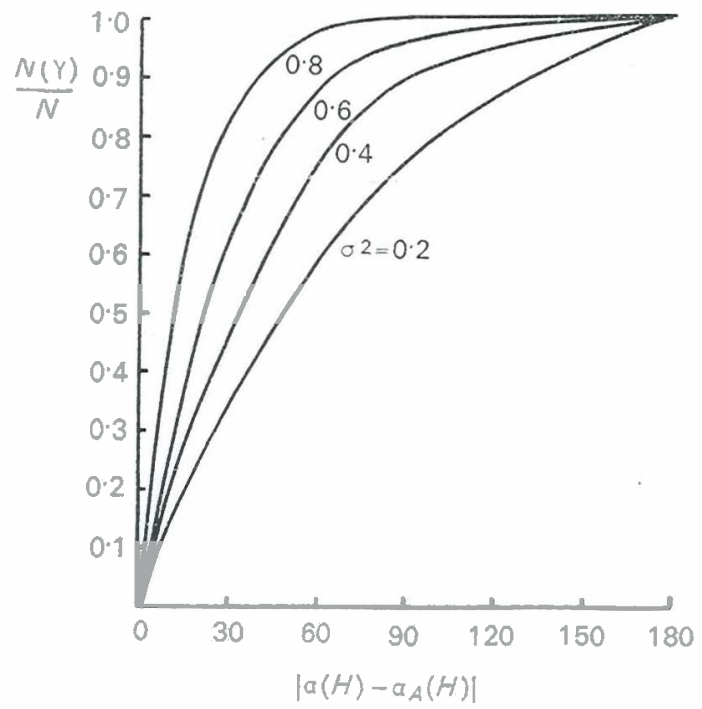

Fig. 1. Cumulative distribution of $\left|\alpha(H)-\alpha_{A}(H)\right|$ for one anomalous scatterer in the unit cell. ber and the arrangement of the anomalous scatterer in the unit cell. For subsequent discussion we shall consider the case of one anomalous scatterer in the unit cell. The distribution of $\left|\alpha(H)-\alpha_{A}(H)\right|$ for various values of $\sigma^{2}$ is shown in Fig. 1. It is seen that the fraction of the total refiexions having $\left|\alpha(H)-\alpha_{A}(H)\right| \leq \pi / 2$ is large and increases with increasing value of $\sigma^{2}$.

However, there is a small fraction of the total number of reffexions with $\left|\alpha(H)-\alpha_{A}(H)\right|>\pi / 2$ and the phases are given by equation (4). If we follow the above mentioned method of resolving the phase ambiguity, i.e. use equation (3) for the calculation of phases, the phases of such reflexions will be wrong, the error being $|2 \theta|$. It must be noted that this error is inherent in the method of resolving the phase ambiguity. Since the number of such reflexions is small, we shall omit them in the present analysis.

\section{The error in the phases}

Let the scale factor* $k$ be such that the observed structure amplitudes $\left|F_{0}(H)\right|$ are related to those on the absolute scale by $\left|F_{0}(H)\right|=k|F(H)|$. Thus $\Delta F_{0}^{2}=k^{2} \Delta F^{2}$ and $\left|F_{0}^{\prime}(H)\right| \simeq k\left|F^{\prime}(H)\right|$ because $\left|F_{A}^{\prime \prime}(H)\right|^{2}$ is usually small compared with $\frac{1}{2}\left[\left.F(H)\right|^{2}+|F(\bar{H})|^{2}\right]$. With wrongly scaled data equation (2) gives

$$
\begin{aligned}
\cos \theta^{\prime} & =\Delta F_{o}^{2} / 4\left|F_{0}^{\prime}(H)\right|\left|F_{A}^{\prime \prime}(H)\right| \\
& =k \Delta F^{2} / 4\left|F^{\prime}(H)\right|\left|F_{A}^{\prime \prime}(H)\right|
\end{aligned}
$$

or

$$
\cos \theta^{\prime}=k \cos \theta \text {. }
$$

Let $\varphi=\theta^{\prime}-\theta$. Thus $\varphi$ is the error introduced in $\theta$.

The phase calculated with this is given by

$$
\begin{aligned}
\alpha_{k}(H) & =\alpha_{A}(H)+\pi / 2-\theta^{\prime} \\
& =\alpha(H)-\varphi .
\end{aligned}
$$

For all values of $k$ less than unity equation (5) is well defined. It is readily seen that $\varphi$ is zero at $\theta=\pi / 2$ for all values of $k$. This is expected because in such a case the reflexion has the heavy atom phase. As $\theta$ decreases from $\pi / 2$ to 0 or increases from $\pi / 2$ to $\pi$, $|\varphi|$ increases. $\varphi$ is positive if $0<\theta<\pi / 2$ and negative if $\pi / 2<\theta<\pi$.

However, if $k>1$, equation (5) is defined only when $|k \cos \theta| \leq 1$. Thus if $k=2$, all cases with $\pi / 3 \leq \theta \leq 2 \pi / 3$ can be described by equation (5). In cases $0 \leq \theta<\pi / 3$ and $2 \pi / 3<\theta \leq \pi$, one gets a value of $\left|\cos \theta^{\prime}\right|$ exceeding unity. This result, though inadmissible, is nevertheless important in practice as it readily suggests that $k>1$.

Hall \& Maslen (1965) have reported the occurrence of such cases in their work. They have tried three procedures for treating such reflexions. One is to set $\theta^{\prime}=0$ if $\cos \theta^{\prime}>1$. The other two are either to omit

* The scale factor defined in this manner is the reciprocal of the commonly defined scale $K=\Sigma\left|F_{d}\right| /|\Sigma| F_{0} \mid$. 
such reflexions completely or to put the phase of the reflexion equal to the heavy atom phase. Setting $\theta^{\prime}=0$ is reported to have provided most reliable results. Let us examine in a specific case $k=2$, the errors introduced in the phases if one adopts the procedure of setting $\theta^{\prime}=0$ when $\cos \theta^{\prime}>1$. For reflexions with $\pi / 3 \leq \theta \leq 2 \pi / 3$, the errors are given by equation (5). Fig. 2 shows a plot of $\varphi$ versus $\theta$. The error introduced in the range $0 \leq \theta<\pi / 3$ varies linearly from 0 to $\pi / 3$. Thus the procedure of setting $\theta^{\prime}=0$ in the case $\cos \theta^{\prime}>1$ leads to correct results when $\theta^{\prime}$ is close to zero. It must be noted that for $2 \pi / 3<\theta \leq \pi, \cos \theta^{\prime}<-1$. No mention* has been made by Hall \& Maslen (1965) of such cases. If the procedure of setting $\theta^{\prime}=0$ is uniformly adopted, the error increases from $2 \pi / 3$ to $\pi$ in the range $2 \pi / 3<\theta \leq \pi$. However, a logical extension of the procedure of setting $\theta^{\prime}=0$ for $\cos \theta^{\prime}>1$ would be to set $\theta^{\prime}=\pi$ if $\cos \theta^{\prime}<-1$. In such cases the error varies linearly from $\pi / 3$ to 0 as $\theta^{\prime}$ changes from $2 \pi / 3$ to $\pi$.

Thus, trying to adopt any procedure such as setting $\theta^{\prime}=0$ or its other equivalent would mean to rely too much on the scale factor which is liable to large errors. A better procedure is to scale down the data so that the largest $\left|\cos \theta^{\prime}\right|$ value encountered is reduced to unity. $\left|\cos \theta^{\prime}\right|>1$ may also arise owing to the errors in the measurement of intensities but such cases will be few. Frequent occurence of such cases must be taken as a positive indication of a high scale factor.

Fig. 3 shows a plot of $\varphi$ versus $(\pi / 2-\theta)$ for $k=0.5$, 1.5 and 2.0 . It is seen that $\varphi$, the error introduced in $\theta$ and hence in the phases is large for large values of $(\pi / 2-\theta)$.

\section{The electron density function}

We have already seen that equation (5) is well defined for all values of $\theta$ if $k \leq 1$ and the phases are given by equation (6). If $k>1$ the phases are given by equation (6) only for reflexions with $|k \cos \theta| \leq 1$. Let the number of such reflexions be $N(k)$. For the remaining $N-N(k)$ reflexions, $N$ being the total number of reflexions, $|k \cos \theta|>1$ and $\alpha_{k}(H)$ depends on how such reflexions are treated. If we follow the scheme of setting $\theta^{\prime}=0$ or $\pi$ according as $\cos \theta^{\prime}>1$ or $\cos \theta^{\prime}<-1$, then

$$
\alpha_{k}(H)=\alpha_{A}(H)+m \pi / 2,
$$

where $m=1$ or -1 in the two cases. Thus the electron density $\varrho_{k}(\mathbf{r})$ computed with wrongly scaled data is given by

$$
\begin{aligned}
& \varrho k(\mathbf{x})= \sum_{H}^{N(k)} k\left|F^{\prime}(H)\right| \exp \{i[\alpha(H)-\varphi]\} \exp \{-2 \pi i \mathbf{H}, \mathbf{r}\} \\
&+\sum_{H}^{N-N(k)} k\left|F^{\prime}(H)\right| \exp \left\{i\left[\alpha_{A}(H)+m \frac{\pi}{2}\right]\right\} \\
& \times \exp \{-2 \pi i \mathbf{H} \cdot \mathbf{r}\} .
\end{aligned}
$$

* According to a private correspondence with Dr S. R. Hall, cases with $\cos \theta^{\prime}<-1$ were observed and $\theta^{\prime}=\pi$ was used in such cases.
Obviously $N(k)=N$ for $k \leq 1$ and the second term exists only for $k>1$.

From equation (5) we have

or

$$
\cos (\theta+\varphi)=k \cos \theta \text { for }|k \cos \theta| \leq 1 \text {, }
$$

$$
=(1-k) \gamma+\frac{k\left(1-k^{2}\right)}{6} \gamma^{3}+\frac{k\left(1-k^{2}\right)\left(9 k^{2}-1\right)}{120} \gamma^{5}+\ldots
$$

where $\gamma=\pi / 2-\theta$. Thus

Let us introduce a function $s(\gamma)$ such that $\varphi=\gamma s(\gamma)$

$$
s(\gamma)=\varphi / \gamma=(1-k)+\frac{k\left(1-k^{2}\right)}{6} \gamma^{2}
$$

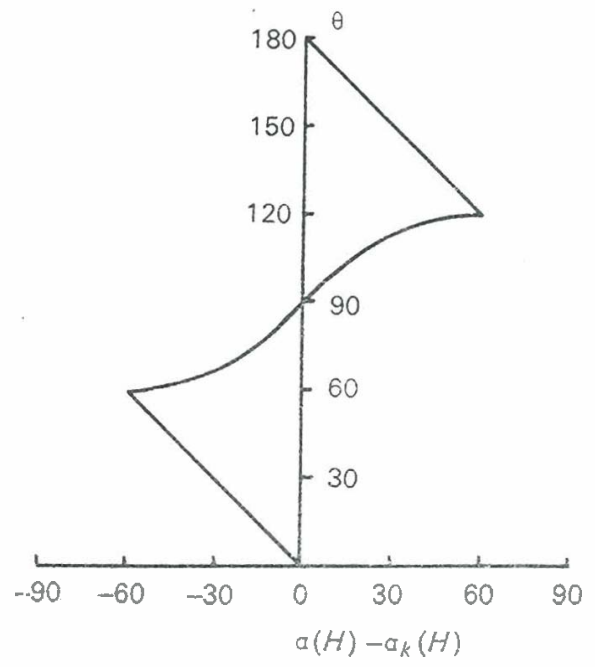

Fig. 2. Errors introduced in the phases as a function of $\theta$ for $k=2$.

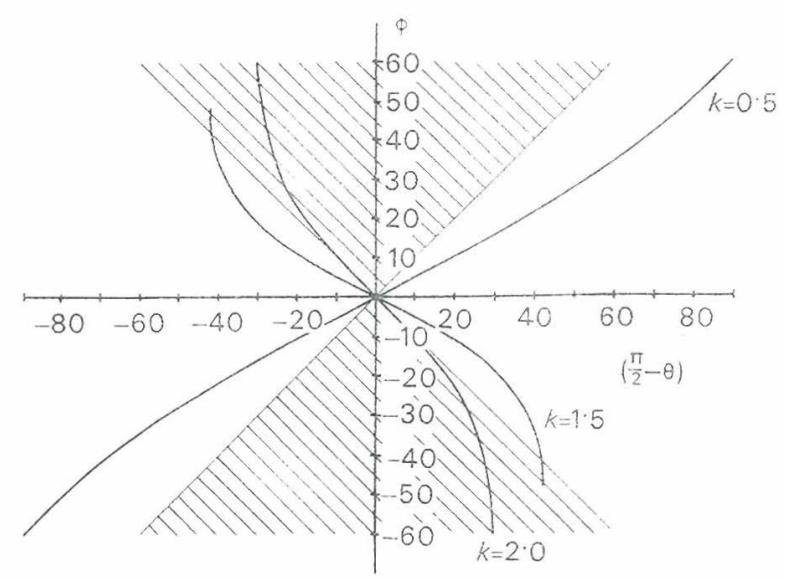

Fig. 3. Plot of $\varphi$ versus $(\pi / 2-\theta)$ for various values of $k$. 
or

where

$$
s(\gamma)=(1-k)+f(\gamma)
$$

$$
f(\gamma)=\frac{k\left(1-k^{2}\right) \gamma^{2}}{6}+\frac{k\left(1-k^{2}\right)\left(9 k^{2}-1\right) \gamma^{4}}{120}+\ldots
$$

Now the following identity can be readily shown:

$$
\begin{aligned}
\exp [-i \varphi] & =\exp [-i \gamma s(\gamma)] \\
& =[1-s(\gamma)]+s(\gamma) \exp [-i \gamma]+|\delta(\gamma)| \exp [i \varepsilon(\gamma)],
\end{aligned}
$$

where

$$
\delta(\gamma) \cos \varepsilon(\gamma)=[\cos \varphi-s(\gamma) \cos \gamma]-[1-s(\gamma)]
$$

and

$$
\delta(\gamma) \sin \varepsilon(\gamma)=[s(\gamma) \sin \gamma-\sin \varphi]
$$

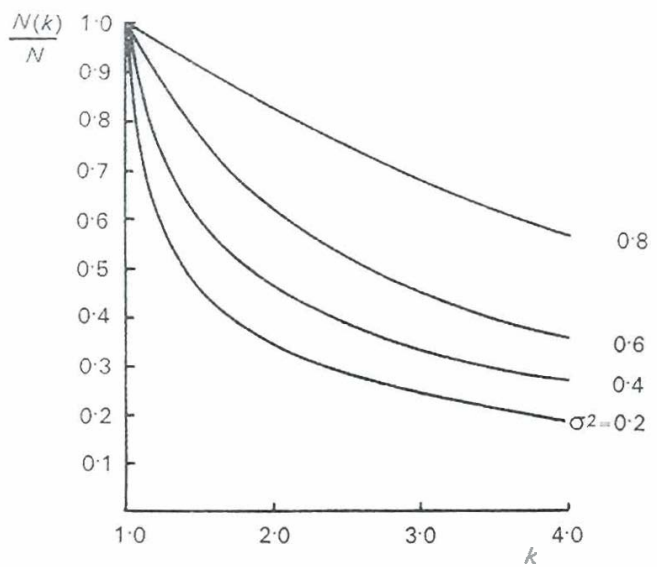

Fig. 4. Plot of $N(k) / N$ versus $k . N(k)$ is the number of reflexions for which $|k \cos \theta| \leq 1$. The curves are deduced from Fig. 1 .

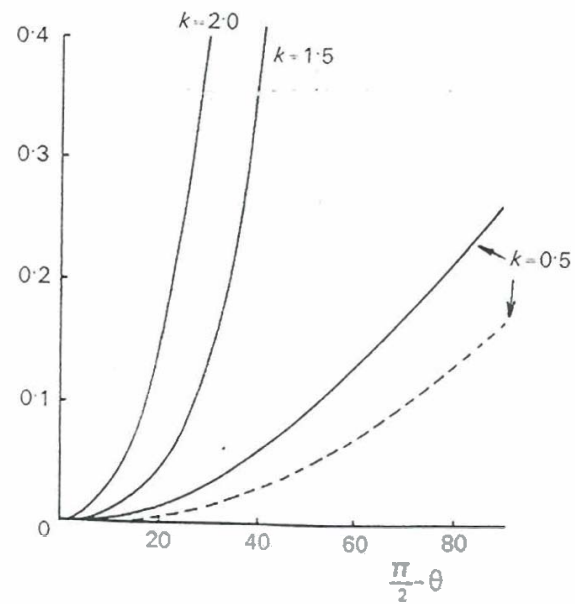

Fig. 5. Plots of $|\delta(y)|$ (full line) and $|f(y)|$ (broken line) versus $|\gamma|$. The $|f(\gamma)|$ plot falls close to the $|\delta(\gamma)|$ plot for $k=1.5$ and 2.
On substituting the value of $s(y)$ from equation $9(a)$ in equation ( 10$)$ we have,

$$
\begin{aligned}
& \exp [-i \varphi]=k-f(\gamma) \\
& \quad+[(1-k)+f(\gamma)] \exp [-i \gamma]+|\delta(\gamma)| \exp [i \varepsilon(\gamma)] .
\end{aligned}
$$

On substituting the value of $\exp [-i \varphi]$ from equation (13) in equation (7) we have,

$$
\begin{aligned}
\varrho_{k}(\mathbf{r})= & k^{2} \varrho_{O} k(\mathbf{r})+k(1-k) \varrho_{A k}(\mathbf{r}) \\
& +k\left[\varrho_{A k}^{\prime}(\mathbf{r})-\varrho_{o k}^{\prime}(\mathbf{r})\right]+k Q^{\prime \prime}(\mathbf{r})+k Q^{\prime \prime \prime}(\mathbf{r})
\end{aligned}
$$

where

$$
\begin{aligned}
& \varrho_{O k}(\mathbf{r})=\sum_{H}^{N(k)}\left|F^{\prime}(H)\right| \exp [i \alpha(H)] \exp [-2 \pi i \mathbf{H}, \mathbf{r}] \\
& \varrho_{A k}(\mathbf{r})=\sum_{H}^{N(k)}\left|F^{\prime}(H)\right| \exp \left[i \alpha_{A}(H)\right] \exp [-2 \pi i \mathbf{H}, \mathbf{r}] \\
& \varrho_{A k}^{\prime}(\mathbf{r})=\sum_{H}^{N(k)}\left|F^{\prime}(H)\right| f(\gamma) \exp \left[i \alpha_{A}(H)\right] \exp [-2 \pi i \mathbf{H}, \mathbf{r}] \\
& \varrho_{a k}^{\prime}(\mathbf{r})=\sum_{H}^{N(k)}\left|F^{\prime}(H)\right| f(\gamma) \exp [i \alpha(H)] \exp [-2 \pi i \mathbf{H}, \mathbf{r}] \\
& Q^{\prime \prime}(\mathbf{r})=\sum_{H}^{N(k)}\left|F^{\prime}(H)\right||\delta(\gamma)| \exp \{i[\alpha(H)+\varepsilon(\gamma)]\} \\
& \quad \times \exp [-2 \pi i \mathbf{H}, \mathbf{r}] \\
& \varrho^{\prime \prime \prime}(\mathbf{r})=\sum_{H}^{N}\left|F^{\prime}(H)\right| \exp \left\{i\left[\alpha_{A}(H)+m \pi / 2\right]\right\} \\
& \times \exp [-2 \pi i \mathbf{H} . \mathbf{r}]
\end{aligned}
$$

The significance of these different syntheses may be described in the following way.

$\varrho_{\circ k}(\mathbf{r})$ - and $\varrho_{A k}(\mathbf{r})$-synthesis

Qok $(\mathbf{r})$ includes all the reflexions for $k \leq 1$. Thus it represents a synthesis which employs the correct phases for all the reflexions but for a few for which $\left|\alpha(H)-\alpha_{A}(H)\right|>\pi / 2$. Therefore, $\varrho_{o k}(\mathbf{r})$ for $k \leq 1$, simply denoted by $\varrho_{o}(\mathbf{r})$ hereinafter, will be very similar to but not the same as $\varrho(\mathbf{r})$, the correct electron density. It must be noted that under the ideal conditions of the measurement of intensities and scaling we shall have only $Q_{o}(\mathbf{r})$. The difference between $\varrho_{0}(\mathbf{r})$ and $\varrho(\mathbf{r})$ is inherently due to the method of resolving the phase ambiguity.

Further it is seen that the number of reflexions with $\left|\alpha(H)-\alpha_{A}(H)\right| \leq \pi / 2$ decreases as $\sigma^{2}$ decreases. Thus for very small value of $\sigma^{2}$ the resemblance between $Q_{0}(\mathbf{r})$ and $\varrho(\mathbf{r})$ may become poor. Structures with $\sigma^{2}-$ values as low as 0.17 and 0.13 . [Factor Vla by Dale, Hodgkin \& Venkatesan (1963) and ' $O$ ' monoacid of $B_{12}$ by Nockolds (1966)] have been solved by this method. In each case $\varrho_{0}(r)$ is very similar to $\varrho(\mathbf{r})$. We shall, therefore, take $Q_{0}(\mathbf{r})$ to be the same as $Q(\mathbf{r})$.

For the case $k>1$, the number, $N(k)$ of the reflexions included in $\varrho_{o} k(r)$ depends on the value of $k$ and the distribution of $\left|\alpha(H)-\alpha_{A}(H)\right|$. Fig. 4 shows a plot 
$N(k) / N$ reersus $k$ for various values of $\sigma^{2}$. For any value of $\sigma^{2}, N(k)$ decreases with increasing value of $k$. Further for a given error in scale factor $N(k)$ decreases with decreasing value of $\sigma^{2}$. Thus as the error in scale factor increases $N(k)$ decreases and $Q_{0 k}(\mathbf{r})$ loses the features of the correct electron density. However, if $k$ is not large $N(k) \simeq N$ and $\varrho_{o k}(\mathbf{r})$ is similar to the correct electron density. Thus largest value of $k$ that can be permitted without spoiling $\varrho_{o k}(\mathbf{r})$ appreciably will depend on the value of $\sigma^{2}$.

A similar explanation holds for $Q_{A k}(\mathbf{x})$. In the case $k \leq 1, \varrho_{A k}(\mathbf{r})$ represents the synthesis based on the heavy atom phases which is known to contain some features of the correct electron density. For $k>1$, $\varrho_{A k}(\mathbf{r})$ includes only $N(k)$ terms and gradually loses the features of the structure as $k$ increases.

$\varrho_{o k}^{\prime}(\mathbf{r})$ - and $\varrho_{A k}^{\prime}(\mathbf{r})$-sy'nthesis

$\varrho_{o k}^{\prime}(\mathbf{r})$ employs the correct phases while the structure amplitudes are modulated by $f(\gamma)$ defined by equation $(9 b) . f(y)$ is positive or negative according as $k<1$ or $k>1$. A plot of $|f(\gamma)|$ versus $\gamma$ (Fig. 5) indicates that $|f(\gamma)|$ is small for small values of $\gamma$. Further it is seen from Fig. I that a large fraction of the total reflexions lies in the small $|\gamma|$ range. Thus most reflexions have a small $|f(\gamma)|$ value. This is made clear in Fig. 6, which shows a plot of $|N(\gamma)| / N$ versus $|f(\gamma)|, N(\gamma)$ being the number of reflexions with $|y|$ values in the range 0 to $|y|$. For $k=0.5$ and $\sigma^{2}=0.4$ nearly $80 \%$ of the reflexions have $|f(\gamma)| \leq 0 \cdot 10$. For $k=1 \cdot 5,45 \%$ of the reflexions have $|f(\gamma)| \leq 0 \cdot 10$. Thus we see that for most reflexions the amplitude terms are suppressed by $f(\gamma)$ modulation. Further, $f(\gamma)$-modulation introduces a certain amount of random character in the amplitudes. Hence $\varrho_{o k}^{\prime}(\mathbf{r})$ is similar to the 'random synthesis' (Srinivasan, 1961) which employs the correct phases but randomly permuted amplitudes. A 'random synthesis' is known to contain the peaks at atomic sites of the structure. Thus $\varrho_{o k}^{\prime}(\mathbf{r})$ contains peaks at atomic sites but the peak strength will be small owing to the small amplitudes used in the synthesis.

A similar interpretation holds for $Q_{A K}^{\prime}(\mathbf{r})$.

However, if we assume that $\alpha(H) \simeq \alpha_{A}(H)$, e.g. in the case where $\sigma^{2}$ is large, then $\varrho_{o k}^{\prime}(\mathbf{r}) \simeq Q_{A k}^{\prime}(\mathbf{r})$ and the term $\left[\varrho_{A k}^{\prime}(\mathbf{r})-\varrho_{o k}^{\prime}(\mathbf{r})\right]$ in equation (14) is nearly zero. If the $\sigma^{2}$ value is small or the heavy atoms are centrosymmetricaliy related, $\varrho_{0 k}^{\prime}(\mathbf{x})$ and $\varrho_{A K}^{\prime}(\mathbf{r})$ will be quite different but as discussed earlier, both the terms will be small.

\section{$\varrho^{\prime \prime}(\mathbf{r})$-synthiesis}

The amplitudes and the phases in $Q^{\prime \prime}(\mathbf{r})$ are $|\delta(y)| F^{\prime}(H) \mid$ and $x(H)+\varepsilon(\gamma)$ respectively. A plot of $|\delta(\gamma)|$ versus $|\gamma|$ (Fig. 5) indicates that $|\delta(\gamma)|$ is similar to $|f(\gamma)|$ in nature as well as in magnitude. Thus $|\delta(\gamma)|-$ modulation suppresses most amplitude terms while $\varepsilon(\gamma)$ disturbs the phases. As a result $Q^{\prime \prime}(\mathbf{r})$ hardly contains any features of the structure and provides only a small background.

\section{$Q^{\prime \prime \prime}(\mathbf{r})$-synthesis}

By definition $Q^{\prime \prime \prime}(r)=0$ for $k \leq 1$. As discussed earlier the convention of setting $\theta^{\prime}=0$ or $\pi$ according as $\cos \theta^{\prime}>1$ or $\cos \theta^{\prime}<-1$ leads to correct values of the phases only for $\theta$ values close to 0 or $\pi$. However in actual cases there are not many reflexions with $\theta$ values close to 0 or $\pi$. For example, in the case of a structure with one heavy atom and $\sigma^{2}=0.6$, only $2 \%$ of the reflexions will have $\theta$ values in the range $0-10^{\circ}$ and $170-180^{\circ}$ If $k=1.5$ in this case, nearly $24 \%$ of the reflexions wil have $\left|\cos \theta^{\prime}\right|>1$. Thus most of these will have wrong phases and therefore $Q^{\prime \prime \prime}(\mathbf{r})$ will mainly provide background.

\section{Discussion}

Thus we see that the electron density computed with wrongly scaled data can be expressed as the sum of five or six component syntheses [equation (14)] accord-

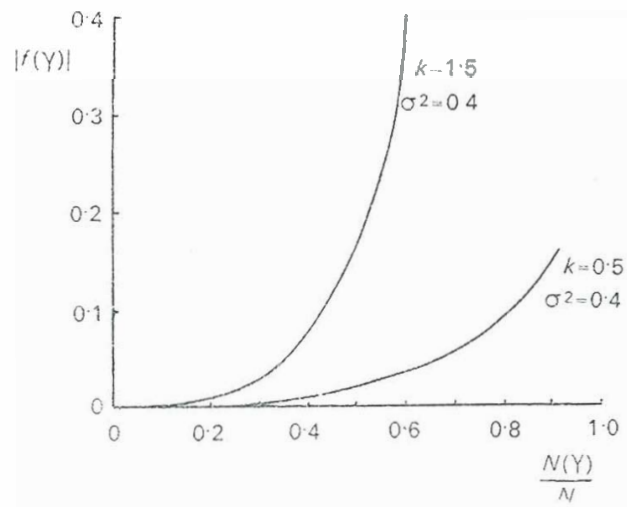

Fig.6. Plot of $|f(y)|$ versus $N(\gamma) / N$. The curves are deduced from Figs. (1) and (5).

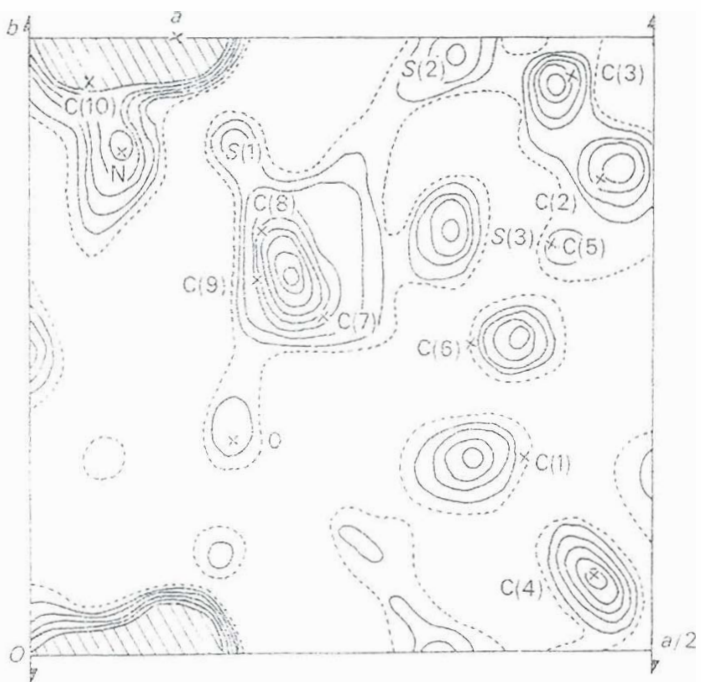

Fig. 7. $e_{0}(\mathbf{r})$. The crosies indicate the correct positions of the atoms. The contout's are from $1.5 \mathrm{e} . \AA^{-2}$ (dotted line) at intervals of $0.5 \mathrm{e} . \mathrm{A}^{-2}$. The shaded region is more than 4 e. $\AA^{-2}$ 
ing as the data are on a scale lower or higher than

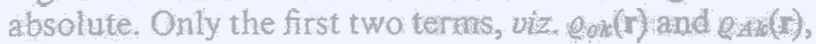
contain the features of the structure and remaining terms contribute mainly to the backgrownd, which increases with increasing value of k. Retaining only the first two terms in equation (14) we have

$$
\varrho_{k}(\mathbf{r}) \simeq k^{2} \varrho_{0 k}(\mathbf{r})+k(1-k) \varrho_{A}(\mathbf{r}) .
$$

For $k \leq 1, \varrho_{0}(\mathbf{r})=\varrho_{0}(\mathbf{r})$ and $Q_{A k}(\mathbf{r})=\varrho_{A}(\mathbf{r})$. When $k>1$ and the error is not large, $\varrho_{o k}(\mathbf{r}) \simeq \varrho_{0}(\mathbf{r})$ and $Q_{A k}(r) \simeq Q_{A}(r)$. Thus equation (15) can be written as

$$
\varrho_{k}(\mathbf{r}) \simeq k^{2} \varrho_{0}(\mathbf{r})+k(1-k) \varrho_{A}(\mathbf{r})
$$

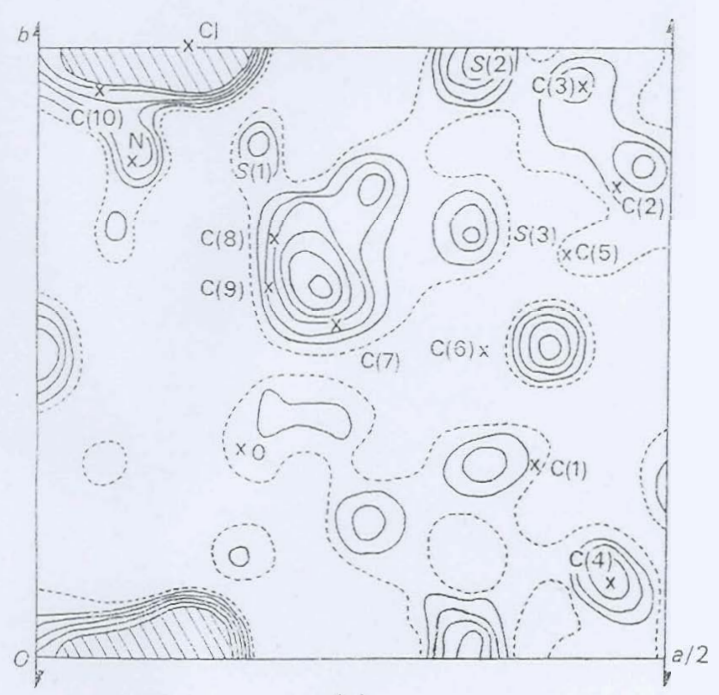

(a)

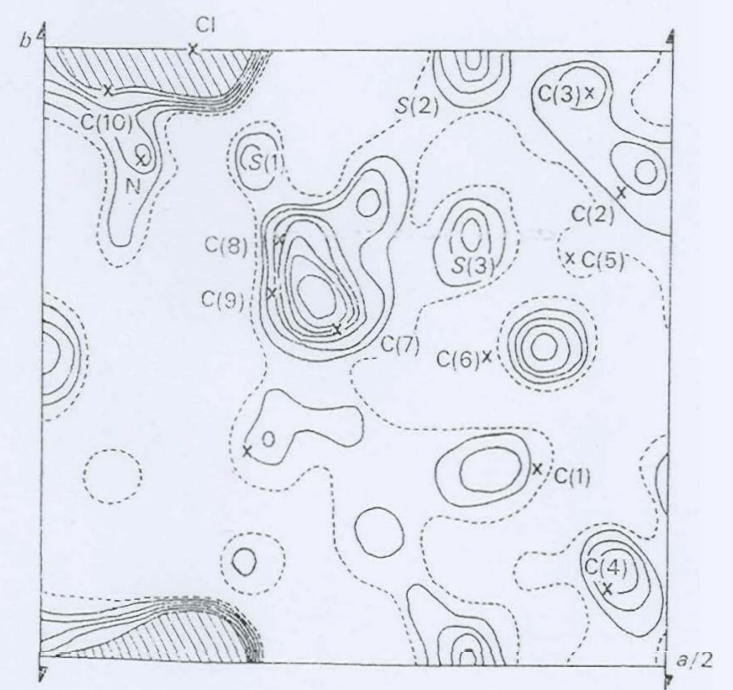

(b)

Fig. 8. (a) $e_{k}(r) / k$ with $k=0.5$. (b) Plot of $0.5[0 .(r)+Q A(z)]$. The contours are drawn from $1.5 e_{.} \AA^{-2}$ at intervals of $0.5 \mathrm{e} . \AA^{-2}$. The shaded region is more than $4 \mathrm{e} . \AA^{-2}$.
$Q_{k}(\mathbf{r})$ is a superposition of the correct electron density (scale $k^{2}$ ) and the synthesis based on the heavy atom phases. (scale $k-k^{2}$ ). For small values of $k$, the first term is small and $\varrho_{n}(r)$ is no better than $e_{A}(\mathrm{r})$. $\varrho_{A}(\mathrm{r})$ normally contains some features of the structure. However it may also contain some spurious peaks or some of the correct peaks may be absent. Besides this some peaks may be slightly shifted from the correct atomic positions. We shall not consider such shift of peaks and broadly classify the peaks encountered in $\varrho_{0}(\mathrm{r})$ and $\varrho_{A}(\mathbf{r})$ as follows:

(a) The most commonly encountered peaks are those which are common to both $\varrho_{0}(\mathbf{r})$ and $Q_{A}(\mathbf{r})$. Thus if $\varrho_{0}(\mathbf{r})$ and $\ell_{A}(\mathbf{r})$ contain peaks of strength $S_{O}$ and $S_{A}$ respectively at a common position $\mathbf{r}$, then $\varrho_{k}(\mathbf{r})$ will contain at $r$ a peak of strength

$$
S_{k} \simeq k^{2} S_{0}+k(1-k) S_{A} \text {. }
$$

If we assume that $S_{0} \simeq S_{A}=S$ then $S_{k} \simeq k S$. This is the strength which will be obtained if amplitudes $k\left|F^{\prime}(H)\right|$ and the correct phases are used to compute the Fourier series.

(b) Often encountered peaks are those which are present only in $\varrho_{0}(r)$ and not in $\varrho_{A}(\mathbf{r})$. Such peaks are of interest in structure analysis as they are correct but do not appear in $\varrho_{A}(\mathbf{r})$. The corresponding peak in $\varrho k(\mathbf{r})$ will have a strength $k^{2} S_{0}$. If $k<1$, such peaks have strength less than their correct value $S_{0}$. If $k>1$, these peaks come up in $\varrho_{k}(\mathbf{r})$ with strength which is more than $S_{0}$.

(c) Another type of peak is present only in $\varrho_{A}(\mathbf{r})$ but not in $\varrho_{0}(\mathbf{r})$. Such peaks are obviously spurious. The corresponding peak in $Q_{k}(\mathbf{r})$ will have a strength $k(1-k) S_{A}$. Thus such peaks are positive for $k<1$ and negative for $k>1$.

An interesting case arises when the heavy atom arrangement has a centre of symmetry. This is so, for example, in the case of space group $P 2_{1}$. The synthesis based on the heavy atom phases contains a spurious mirror symmetry. Thus corresponding to every $(a)$-type peak at $\mathbf{r}_{a}$ in $\varrho_{A}(\mathbf{r})$, there occurs a peak of equal strength at $\mathbf{r}_{m} ; \mathbf{r}_{a}$ and $\mathbf{r}_{m}$ are related by spurious mirror symmetry. Obviously the peak at $\mathbf{r}_{m}$ belongs to type (c).

If the peak strengths at $\mathbf{r}_{a}$ and $\mathbf{r}_{m}$ in $\varrho_{k}(\mathbf{r})$ be $S(k)$ and $S_{m}(k)$ respectively, then it can be easily shown from equation (16) that,

$$
\frac{S_{m}(k)}{S(k)} \simeq \frac{(1-k) S_{A}}{k S_{o}+S_{A}(1-k)} .
$$

If $S_{A} \simeq S_{0}$ then

$$
\frac{S_{m}(k)}{S(k)} \simeq(1-k)
$$

Clearly for $k<1, S_{m}(k)$ is positive and the spurious mirror symmetry which is present in $Q_{A}(r)$ is not completely destroyed in $\varrho_{k}(\mathrm{r})$. If $k>1, S_{m}(k)$ is negative.

In practice it seems more advantageous to have $k>1$, firstly because corresponding to every (c)-type peak in 
$\varrho_{A}(\mathbf{r})$ there is a negative peak in $\varrho_{k}(r)$ and secondly because corresponding to every (b)-type peak in $\varrho_{0}(\mathbf{r})$ there occurs in $\varrho_{k}(\mathbf{r})$ a peak with increased strength. Both these factors are of great importance in actual structure analysis. However, if $k$ is large these advantages may be lost by increase in background.

The results obtained thus far can be explained qualitatively as follows: In the anomalous dispersion method of phase calculation, $\alpha_{A}(H)$ may be regarded as having to be corrected by a factor $(\pi / 2-\theta)$, giving $\alpha(H)$. Thus the difference between $\varrho_{0}(\mathbf{r})$ and $\varrho_{A}(\mathbf{r})$ which employ $\alpha(H)$ and $\alpha_{A}(H)$ respectively is due to the correction term $(\pi / 2-\theta)$. Thus the correction term suppresses the spurious peaks in $\varrho_{A}(\mathbf{r})$ and builds up the correct ones. It can be easily shown from equation (5) that the magnitude of the correction term $(\pi / 2-\theta)$ is always underestimated if $k<1$. As a result $\varrho k(\mathbf{r})$ will be better than $\varrho_{A}(\mathbf{r})$ but the spurious peaks in $\varrho_{A}(\mathbf{r})$ will not be completely suppressed, nor will the correct peaks be adequately strengthened. For $k>1$, the magnitude of the correction term is overestimated. Thus the improvement which is brought about by the correction term is overdone. As a result, the spurious peaks in $\varrho_{A}(\mathbf{r})$ which are suppressed in $\varrho_{0}(\mathbf{r})$ will be rendered negative in $\varrho_{k}(\mathbf{r})$. On the other hand the correct peaks which are not present in $\varrho_{A}(\mathbf{r})$ will be over strengthened in $\varrho_{k}(\mathbf{r})$.

This rather simple analysis does not give any quantitative idea of the background provided by the other terms in equation (14). It can be stated qualitatively that for a given structure the background increases with the increasing value of $k$. Further, two structures with different $\sigma^{2}$ values will have different amounts of background for a given error in the scale factor; the structure with larger $\sigma^{2}$ will have smaller background.

\section{Experimental verification}

In order to verify the results obtained in this paper, the projection data on ephedrine hydrochloride (Phillips, 1954) have been used.

From the knowledge of $\alpha(H)$ and $\alpha_{A}(H), \alpha_{k}(H)$ for $k=0.5,1.0$ and 1.5 have been calculated. Although experimentally observed anomalous dispersion data are available (Ramachandran \& Raman, 1956) and could have been used for the calculation of $\alpha_{k}(H)$, the following procedure was adopted in order to eliminate the errors in the measurement of intensities:

$$
\alpha_{k}(H)=\alpha_{A}(H)+\gamma^{\prime},
$$

where

If $k \sin \gamma>1$

$$
\begin{aligned}
& y^{\prime}=\sin ^{-1}(k \sin \gamma) \\
& y=\alpha(H)-\alpha_{A}(H) .
\end{aligned}
$$

if $k \sin \gamma<-1$

$$
\alpha_{k}(H)=\alpha_{A}(H)+\pi / 2 ;
$$

$$
\alpha_{k}(H)=\alpha_{A}(H)-\pi / 2 .
$$

If the experimental data are used and the phases are calculated from equations (2) and (3) then $\left|\alpha(H)-\alpha_{A}(H)\right| \leq \pi / 2$. However, in the present example 16 out of 80 reflexions have $\left|\alpha(H)-\alpha_{A}(H)\right|>\pi / 2$. In order to make the above calculations correspond to actual anomalous dispersion calculation, $\gamma$ and $\gamma^{\prime}$ are always chosen between $\pi / 2$ and $-\pi / 2$. If the actual value of $y$ is outside this range $y$ is subtracted from $\pi$ in order to bring $y$ in the desired range. All the computations have been made on a Ferranti Sirius computer. On this computer this is best done by taking $A R C S I N$ $(S I N \gamma)$; which is always given in the range $\pi / 2$ and $-\pi / 2$.

The following computations were made with the use of these phases:

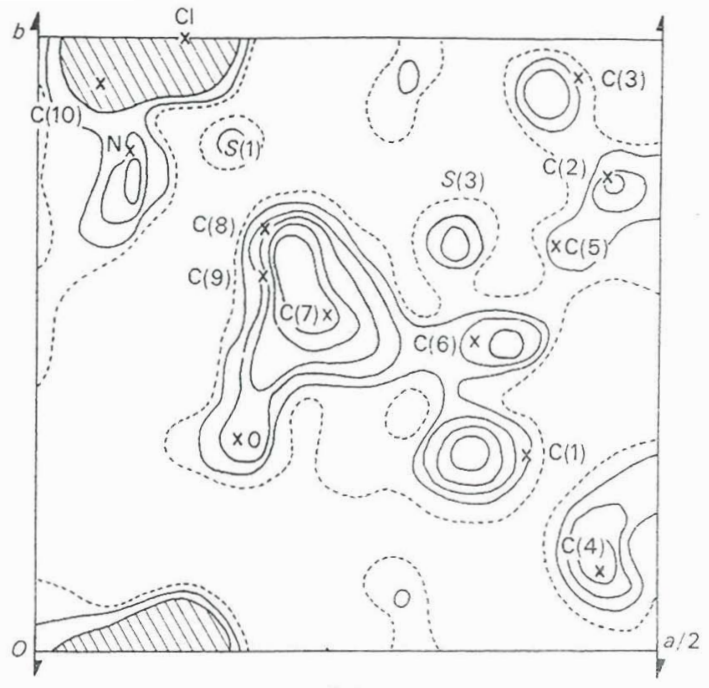

(a)

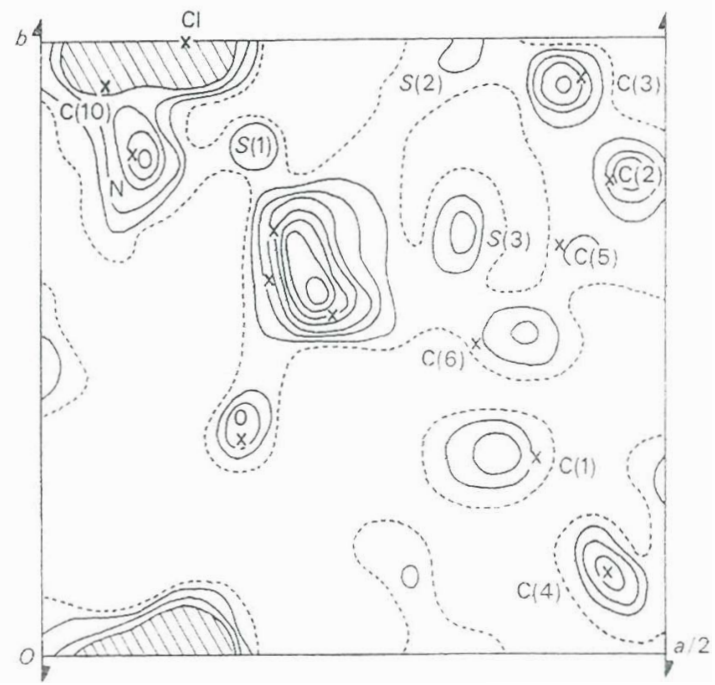

(b)

Fig. 9. (a) $Q k(\mathbf{r}) / k$ with $k=1.5$. (b) A plot of [1.5Q0 $\left.(\mathbf{r})-0.5 Q_{A}(\mathbf{r})\right]$. The contours are drawn from 1.5 e. $\AA^{-2}$ at intervals of $1 \in \AA^{-2}$. The shaded region is more than $5 \mathrm{e} . \AA^{-2}$. 
(i) $\alpha_{k}(\boldsymbol{H})$ for $k=1$ were evaluated and a Fourier synthesis with these phases has been computed (Fig.7). 13 centrosymmetric reflexions of the type $h 00$ have not been included in the summation.

In Fig. 7 all atoms bave come up correctly except $C(1)$ and $C(6)$, which are displaced from their correct positions. After including $h 00$ terms peak $\mathrm{C}(1)$ shifts halfway towards the correct position.

The atoms $C(5)$ and $O$ have not come out with their correct strengths. This is mainly because $h 00$ terms have been omitted. On including these terms both the peaks have the correct strengths.

Three spurious peaks marked $S_{1,} S_{2}$ and $S_{3}$ appear of which all except $S_{1}$ are suppressed on adding $h 00$ terms. $S_{1}$ becomes slightly stronger.

Thus the only difference between $\varrho_{0}(\mathbf{r})$ and $\varrho(\mathbf{r})$ is that the former contains one spurious peak $S_{1}$ and the peaks $C(1)$ and $C(6)$ are slightly displaced from their correct positions.

(ii) $Q_{A}(\boldsymbol{r}) / k$, the synthesis based on the heavy atom phases was computed (not shown in the Figure).

(iii) $\mathrm{ex}(\mathrm{x}) / k$ with $k=0.5$ and 1.5 were computed and are shown in Figs.8(a) and $9(a)$ respectively.

(iv) Fig. 8(b) shows a plot of $\left[0.5 \varrho_{0}(\mathbf{r})+0.5 \varrho_{A}(\mathbf{r})\right]$.

(v) Fig. $9(b)$ shows a plot of $\left[1 \cdot 5 \varrho_{0}(\mathbf{r})-0.5 \varrho_{A}(\mathbf{r})\right]$.

A comparison of Figs. $8(a)$ and $9(a)$ with $8(b)$ and 9 (b) respectively shows that the first two terms, viz. $\varrho_{o k}(\mathbf{r})$ and $\varrho_{A k}(\mathbf{r})$, in equation (14) describe $\varrho_{k}(\mathbf{r})$ satisfactorily.

An analysis of Figs. $8(a)$ and $8(b)$ indicates that the difference between the two maps is a maximum at the $\mathrm{Cl}$ peak, the difference being $0.9 \mathrm{e} . \AA^{-2}$, and nowhere else does the difierence exceed 0.6 e. $\AA^{-2}$. For Fig. $9(a)$ and $(b)$ the difference at the $\mathrm{Cl}$ peak is about 2 e. $\AA^{-2}$ and elsewhere it is less than $1 \cdot 5 \cdot$ e. $\AA^{-2}$.

\section{Conclusion}

The degree of correspondence between the Fourier synthesis with wrong scale and the synthesis of the correct structure is decided jointly by the error in scale and the heavy atom ratio. For the values of heavy atom ratio and the errors in scale normally encountered in practice, the background terms in equation (14) are small and the electron density with wrong scale is described to a good approximation by the superposition of the correct electron density (scale $k^{2}$ ) and the synthesis based on the heavy atom phases (scale $k-k^{2}$ ). This explains the general insensitiveness of the electron density map to the errors in scale factor. However if the error in scale factor is so large that the contributions from the background terms increase considerably, the quality of the electron density map is bound to suffer.

The author is grateful to Dr S. Ramaseshan for his keen interest in the work. Thanks are also due to Dr $\mathrm{K}$. Venkatesan of the University of Madras for many helpful discussions, to Dr S.R. Hall of the University of Western Australia for having so kindly sent the data on MMI and clarified so many points and to the referee for his constructive criticism of the first draft of the paper. The help in computation received from Mr S.Janardhan and other members of the computer division of the National Aeronautical Laboratory is gratefully acknowledged. Thanks are due to Dr S.R. Valluri, Director of the National Aeronautical Laboratory, for his permission to publish this work.

\section{References}

Dale, D., Hodgkin, D. C. \& Venkatesan, K. (1963). Crystallography and Crystal Perfection, p. 237. New York: Academic Press.

GeURTZ, T. J. H. (1963). Thesis, State Univ., Utrecht.

HALl, S. R. \& MAsLeN, E. N. (1965). Acta Cryst. 18, 265.

Nockolds, C. E. (1966). Thesis, Oxford Univ.

Oraya, Y. \& Pepinsky, R. (1956). Phys. Rev. 103, 1645.

Parthasarathy, S. (1965). Acta Cryst. 18, 1028.

Peerdeman, A. F. \& Bijvoet, J. M. (1956). Proc. Koninkl. Ned. Akad. Welenschap. B59, 312.

Prillips, D. C. (1954). Acta Cryst. 7, 159.

RAMACHANDRAN, G. N. \& RAMAN, S. (1956). Curr. Sci. 25, 348.

Srinivasan, R. (1961). Proc. Indian Acad. Sci. A53, 252.

Wilson, A. J. C. (1942). Nature, Lond. 150, 151. 